\title{
The Role of the Gut Barrier Function in Health and Disease
}

\author{
Stelios F. Assimakopoulos ${ }^{\mathrm{a}, \mathrm{d}}$, Christos Triantos ${ }^{\mathrm{b}}$, Ioannis Maroulis ${ }^{\mathrm{c}}$, \\ Charalambos Gogos ${ }^{\mathrm{a}}$
}

The intestinal tract contains the body's largest interface between a person and his or her external environment. The complexity of its function is obvious when thinking that at the same time the intestine must serve two opposite functions; the selective permeability of needed nutrients from the intestinal lumen into the circulation and into the internal milieu in general and, on the other hand, the prevention of the penetration of harmful entities including microorganisms, luminal antigens, and luminal proinflammatory factors. The latter function is known as barrier function [1].

The gut barrier function is comprised by three major lines of defence [2]: 1) The biological barrier, which is made up of normal intestinal flora (gut microbiota) responsible for colonization resistance; 2) The immune barrier, which is composed of gut associated lymphoid tissue (GALT), effector and regulatory T cells, IgA producing B (plasma) cells, group 3 innate lymphoid cells, and, resident macrophages and dendritic cells in the lamina propria; and 3) The mechanical barrier, consisting of the closed-lining intestinal epithelial cells and by the capillary endothelial cells. The epithelial and endothelial cells come into the closest possible contact in the most apical part of the lateral cell membranes ("kissing points") by specific structures named "tight junctions" (TJs), which interconnect the cells and restrict the passage of ions, molecules and cells through the paracellular space $[2,3]$.

The term "bacterial translocation" (BT), was first described by Berg and Garlington in 1979, as the phenomenon of passage of viable bacteria from the gastrointestinal tract through the epithelial mucosa into the lamina propria and then to the mesenteric lymph nodes and possibly other normally sterile organs [4]. This initial definition was later widened to include the translocation of non-viable bacteria or their products, namely pathogen-associated molecular patterns (PAMPs), with main representative the intestinal endotoxin. BT occurs in healthy in-

Manuscript submitted May 31, 2018, accepted June 7, 2018

aDivision of Infectious Diseases, Department of Internal Medicine, University of Patras Medical School, Patras 26504, Greece

${ }^{b}$ Division of Gastroenterology, Department of Internal Medicine, University of Patras Medical School, Patras 26504, Greece

'Department of Surgery, University of Patras Medical School, Patras 26504, Greece

${ }^{\mathrm{d} C o r r e s p o n d e n c e ~ A u t h o r: ~ S t e l i o s ~ F . ~ A s s i m a k o p o u l o s, ~ D i v i s i o n ~ o f ~ I n f e c t i o u s ~}$ Diseases, Department of Internal Medicine, University of Patras Medical School, Patras 26504, Greece. Email: sassim@upatras.gr

doi: https://doi.org/10.14740/gr1053w dividuals in a low rate of $5-10 \%$, serving two main physiological roles; the antigenic exposure of the gut immune system to be prepared for an effective immune response in case of extensive pathogen invasion, and the development of immune tolerance to several microbial antigens of commensal microflora [5-7].

The intestinal barrier is compromised in several disease states leading to an increased level of BT associated with infectious complications and promotion of a systemic inflammatory response that aggravates the pathophysiological consequences of the underlying disease [8-13]. There are three main pathophysiological groups of intestinal barrier failure associated with pathologic conditions:

1) The intestinal barrier failure observed in surgical patients subjected to major operations for diverse reasons (major liver resections, bowel resections for malignancy, bowel transplantation, aortic aneurysm repair). In this group of patients, increased BT is associated with increased postoperative infectious complications [5, 11, 14-17]. The connecting mechanism is translocation of gut-derived pathogens through a dysfunctional mucosal barrier to the mesenteric lymph nodes, the portal vein and the systemic circulation, eventually leading to postoperative infections [18]. Also, this is the mechanism by which the necrotic pancreas becomes infected in patients with severe necrotic pancreatitis [19].

2) The second group includes critically ill patients, severely injured or septic, hospitalized in intensive care units. Increased gut permeability is associated with the development of systemic inflammatory response and multiple organ dysfunction syndrome (MODS) in these patients. However, the connecting pathophysiological link of gut barrier failure and MODS does not seem to be the classical process of BT [18]. Current pathogenetic aspects support the "gut-lymph" theory of sepsis and MODS. According to this theory, microbes and/ or their products, through a dysfunctional gut barrier, first gain access to the intestinal submucosa activating the intestinal immunological system of defence. An intestinal proinflammatory response further aggravates intestinal injury and dangerassociated molecular patterns (DAMPs) are released in the mesenteric lymphatics, carried to the lung and the systemic circulation, stimulating Toll like receptors- 4 and perhaps other pattern recognition receptors (PRR) in a fashion similar to bacteria, thus eventually promoting injurious effects in diverse organs [20]. Therefore, the gut becomes a pivotal proinflammatory organ promoting deleterious effects in even distant organs, through release of DAMPs, without the need of systemic bacterial translocation $[18,20]$.

3) The third group of intestinal barrier dysfunction involves 
stable patients with chronic pathologic conditions that present a low-grade translocation of enteric microbes and immunostimulatory bioproducts from the gut lumen first in the lamina propria and thereafter in the systemic circulation, promoting a chronic immune activation associated with disease progression and/or development of complications and comorbidities from other organs [1, 21, 22]. This intestinal barrier dysfunction group encompasses patients with HIV infection, liver cirrhosis, chronic viral hepatitis $\mathrm{B}$ or $\mathrm{C}$, non-alcoholic steatohepatitis or non-alcoholic fatty liver disease, patients with inflammatory bowel diseases, celiac disease, irritable bowel syndrome, obesity and diverse autoimmune conditions [1, 21-23]. For example, in HIV infection, intestinal barrier dysfunction, BT and chronic immune activation have been associated with cardiovascular, neurocognitive and lymphoproliferative comorbidities, despite effective viral suppression with modern antiretroviral treatment; and in liver cirrhosis intestinal barrier dysfunction has been associated with all of its complications, namely spontaneous bacterial peritonitis, hepatic encephalopathy, hepatorenal syndrome, hepatopulmonary syndrome, variceal bleeding, progression of liver injury and hepatocellular carcinoma [21, 22].

In conclusion, the Hippocratic quote "all disease begins in the gut" seems to be true, over 2,000 years later, for diverse pathological conditions. Our knowledge on the pivotal role of the intestinal barrier and gut microbiota in health and disease has been majorly developed and constitutes currently a scientific field of intense research. Clinicians should not neglect considering this central pathophysiological role of the gut and should apply all necessary preventive measures to protect the integrity of their patients' intestines in diverse intestinal and extra-intestinal diseases. Future research with application of modern systems biology approaches, namely using genomics, transcriptomics and proteomics might lead to specific and potentially individualized pharmacological targets for intervention to control intestinal hyperpermeability.

\section{Conflict of Interest}

None of the authors has any conflict of interest to report that may be related to this submission.

\section{Author Contributions}

All authors contributed equally.

\section{Funding}

No funding by any private or public party was received for this Editorial.

\section{Abbreviations}

TJ: tight junctions; BT: bacterial translocation; PAMPS: pathogen-associated molecular patterns; DAMPs: danger-associ- ated molecular patterns; PRR: pattern recognition receptors; MODS: multiple organ dysfunction syndrome

\section{References}

1. Konig J, Wells J, Cani PD, Garcia-Rodenas CL, MacDonald T, Mercenier A, Whyte J, et al. Human intestinal barrier function in health and disease. Clin Transl Gastroenterol. 2016;7(10):e196.

2. Assimakopoulos SF, Scopa CD, Vagianos CE. Pathophysiology of increased intestinal permeability in obstructive jaundice. World J Gastroenterol. 2007;13(48):6458-6464.

3. Assimakopoulos SF, Papageorgiou I, Charonis A. Enterocytes' tight junctions: From molecules to diseases. World J Gastrointest Pathophysiol. 2011;2(6):123-137.

4. Berg RD, Garlington AW. Translocation of certain indigenous bacteria from the gastrointestinal tract to the mesenteric lymph nodes and other organs in a gnotobiotic mouse model. Infect Immun. 1979;23(2):403-411.

5. Sedman PC, Macfie J, Sagar P, Mitchell CJ, May J, ManceyJones B, Johnstone D. The prevalence of gut translocation in humans. Gastroenterology. 1994;107(3):643-649.

6. Vaishnavi C. Translocation of gut flora and its role in sepsis. Indian J Med Microbiol. 2013;31(4):334-342.

7. Wiest R, Lawson M, Geuking M. Pathological bacterial translocation in liver cirrhosis. J Hepatol. 2014;60(1):197209.

8. Wiest R, Rath HC. Gastrointestinal disorders of the critically ill. Bacterial translocation in the gut. Best Pract Res Clin Gastroenterol. 2003;17(3):397-425.

9. Ambrose NS, Johnson M, Burdon DW, Keighley MR. Incidence of pathogenic bacteria from mesenteric lymph nodes and ileal serosa during Crohn's disease surgery. Br J Surg. 1984;71(8):623-625.

10. Deitch EA. Simple intestinal obstruction causes bacterial translocation in man. Arch Surg. 1989;124(6):699-701.

11. Woodcock NP, Sudheer V, El-Barghouti N, Perry EP, MacFie J. Bacterial translocation in patients undergoing abdominal aortic aneurysm repair. Br J Surg. 2000;87(4):439-442.

12. Assimakopoulos SF, Thomopoulos KC, Patsoukis N, Georgiou CD, Scopa CD, Nikolopoulou VN, Vagianos CE. Evidence for intestinal oxidative stress in patients with obstructive jaundice. Eur J Clin Invest. 2006;36(3):181-187.

13. Assimakopoulos SF, Tsamandas AC, Tsiaoussis GI, Karatza E, Triantos C, Vagianos CE, Spiliopoulou I, et al. Altered intestinal tight junctions' expression in patients with liver cirrhosis: a pathogenetic mechanism of intestinal hyperpermeability. Eur J Clin Invest. 2012;42(4):439446.

14. O'Boyle CJ, MacFie J, Mitchell CJ, Johnstone D, Sagar PM, Sedman PC. Microbiology of bacterial translocation in humans. Gut. 1998;42(1):29-35.

15. MacFie J, O'Boyle C, Mitchell CJ, Buckley PM, Johnstone D, Sudworth P. Gut origin of sepsis: a prospective study investigating associations between bacterial translocation, gastric microflora, and septic morbidity. Gut. 
1999;45(2):223-228.

16. Chin KF, Kallam R, O'Boyle C, MacFie J. Bacterial translocation may influence the long-term survival in colorectal cancer patients. Dis Colon Rectum. 2007;50(3):323330 .

17. MacFie J, Reddy BS, Gatt M, Jain PK, Sowdi R, Mitchell CJ. Bacterial translocation studied in 927 patients over 13 years. Br J Surg. 2006;93(1):87-93.

18. Deitch EA. Gut-origin sepsis: evolution of a concept. Surgeon. 2012;10(6):350-356.

19. Isaji S, Mizuno S, Tabata M, Yamagiwa K, Yokoi H, Uemoto S. Bacterial analysis of infected pancreatic necrosis and its prevention (Symposium 8: Pancreatobiliary infection (IHPBA)). J Hepatobiliary Pancreat Surg. 2003;10(6):419-424.

20. Deitch EA. Gut lymph and lymphatics: a source of factors leading to organ injury and dysfunction. Ann N Y Acad Sci. 2010;1207 Suppl 1(E103-111.

21. Tsiaoussis GI, Assimakopoulos SF, Tsamandas AC, Triantos $\mathrm{CK}$, Thomopoulos KC. Intestinal barrier dysfunction in cirrhosis: Current concepts in pathophysiology and clinical implications. World J Hepatol. 2015;7(17):20582068.

22. Assimakopoulos SF, Dimitropoulou D, Marangos M, Gogos CA. Intestinal barrier dysfunction in HIV infection: pathophysiology, clinical implications and potential therapies. Infection. 2014;42(6):951-959.

23. Assimakopoulos SF, Charonis AS. Uncovering the molecular events associated with increased intestinal permeability in liver cirrhosis: the pivotal role of enterocyte tight junctions and future perspectives. J Hepatol. 2013;59(5):1144-1146. 